\title{
Bright PanSTARRS Nuclear Transients - what are they?
}

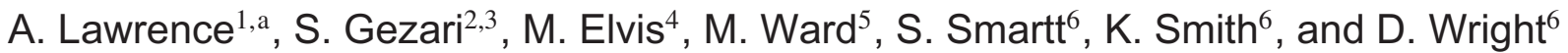 \\ ${ }^{1}$ University of Edinburgh \\ 2 Johns Hopkins University \\ ${ }^{3}$ University of Maryland \\ ${ }^{4}$ Harvard-Smithsonian Center for Astrophysics \\ ${ }^{5}$ University of Durham \\ ${ }^{6}$ Queens University Belfast
}

\begin{abstract}
We present an initial analysis of 49 bright transients occurring in the nuclei of galaxies with no previous known Active Galactic Nucleus (AGN). They have been discovered as part of the PanSTARRs $3 \pi$ survey, and followed up with the Liverpool Telescope. Based on colours, light curve shape, and a small number with optical spectra, these transients seem to fall into three groups. Red/fast transients are nuclear supernovae of various types. Some bright nuclear transients are blue and decay on a timescale of a few months; these may be candidates for tidal disruption events. However most of the events we have found are blue and are either still rising or decaying slowly, on a timescale of years; the few spectra we have show AGN at $\mathrm{z} \sim 1$. We argue that these transients are background AGN microlensed by stars in foreground galaxies by a factor 10-100. Monitoring such events gives us very promising prospects for measuring the structure of AGN and so testing current theories.
\end{abstract}

\section{THE PanSTARRS BRIGHT TRANSIENT SURVEY}

PanSTARRS-1 (PS1) is a $1.8 \mathrm{~m}$ very wide field (7 sq. deg.) telescope in Hawaii which has been surveying the sky repeatedly in grizy since March 2010 (Kaiser et al. 2010). Some of the time is spent observing a set of fixed "Medium Deep Survey (MDS)" fields every few days, but the majority of time is used to survey three quarters of the sky at the rate of $2000 \mathrm{sq} . \mathrm{deg} / \mathrm{nt}-$ the $3 \pi$ survey. The data is processed in Maui, Harvard, and Belfast to produce an automated stream of transients. The brightest transients are found by comparing sources detected in the $3 \pi$ survey with catalogued objects in the Sloan Digital Sky Survey (SDSS), and it these bright transients which we consider here. A prime goal of our programme is the detection of Tidal Disruption events (TDEs). We therefore selected events that were coincident within $0.5^{\prime \prime}$ with a pre-existing object classified in SDSS as a galaxy, with no known AGN activity, and at least 1 mag brighter than the SDSS galaxy.

The PS1 $3 \pi$ imaging does not provide $u$-band measurements, and does not have high enough cadence to properly measure the expected decay timescale of TDEs (weeks-months). We have therefore followed up targets with the Liverpool Telescope at $u g r$, initially with a weekly cadence. So far we do not have a dedicated spectroscopic programme, but some of our objects targets have been serendipitiously observed as part of the related Belfast SN programme.

Here we summarise our initial results. A fuller description is in Lawrence et al. (2012, in preparation).

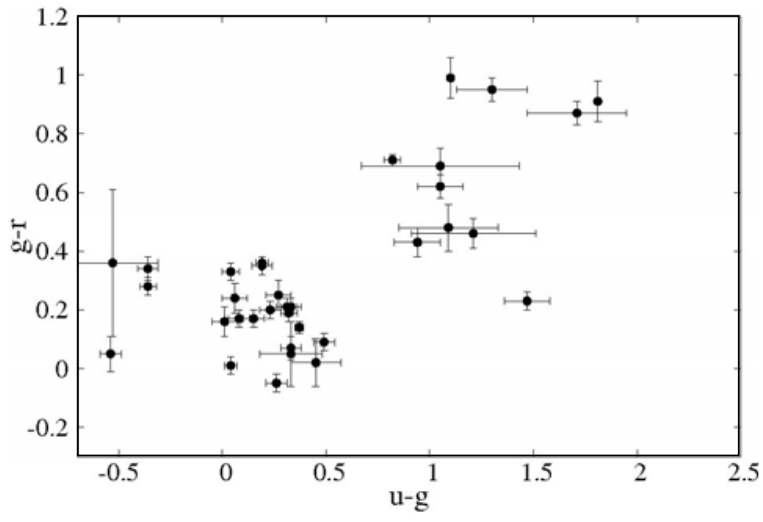

Figure 1. Colours of bright nuclear transients.

\section{PROPERTIES OF BRIGHT NUCLEAR TRANSIENTS}

At the time of giving this talk, we had observed 49 targets between October 2011 and June 2012, around two-thirds of which had enough data for a sensible analysis. The transients concerned typically have $g \sim 19-20$ whereas the pre-existing SDSS galaxies have $g \sim 21-23$, and colours consistent with being normal galaxies at $z \sim 0.1-0.3$. In Fig. 1 we show the $u-g$ vs $g-r$ colours of the transient objects. There are three groups. Red and blue objects divide fairly cleanly at $u-g=0.6$. Objects in the middle (blue) group have colours typical of quasars (e.g. Richards et al. 2001). However there is also a handful of ultra-blue objects, considerably bluer than almost all known quasars.

\section{LIGHT CURVES AND SPECTRA}

In Fig. 2 we show light curves of our bright nuclear transients, with the symbols coloured according the

a e-mail: al@roe.ac.uk

This is an Open Access article distributed under the terms of the Creative Commons Attribution License 2.0, which permits unrestricted use, distribution, and reproduction in any medium, provided the original work is properly cited. 


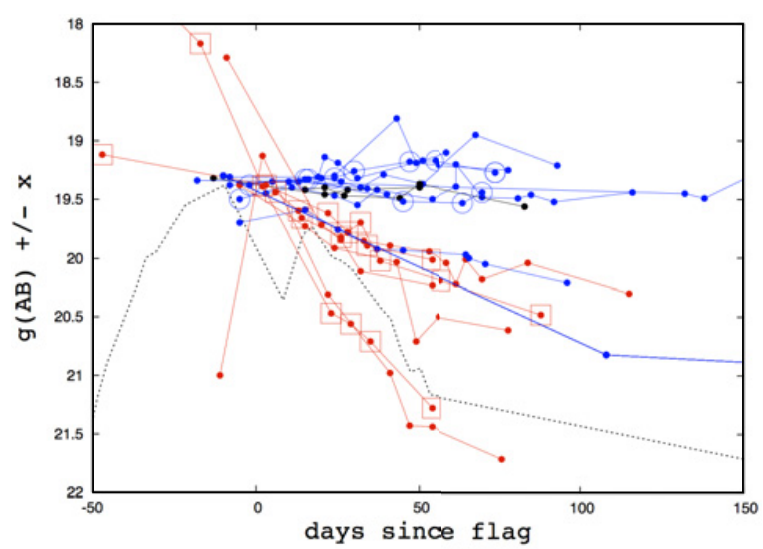

Figure 2. Lightcurves of bright nuclear transients in the gband, arbitrarily normalised for the purposes of illustration. Red symbols are those with $u-g>0.6$. Open symbols indicate objects with available spectra. Open squares are those classified as $\mathrm{SNe}$, and open circles those classified as AGN. The dotted curve is the light curve of the PanSTARRs TDE candidate PS1-10jh.

division at $u-g=0.6$. The red objects all decay reasonably quickly, with two-folding timescales of around a month. Some of the blue objects also decay that fast, but most are much slower, and in many cases consistent with being still rising, with two-folding timescales of very roughly one to a few years. This timescale is much slower than expected for TDEs (e.g. Evans and Kochanek 1989; Lodato et al. 2009).

The PanSTARRS TDE candidate PS1-10jh (Gezari et al. 2012) is also shown on the plot, and is similar to the faster objects. Objects which have spectra taken as part of the Belfast SN programme are marked with open symbols. Those which have been classified as $\mathrm{SNe}$ of various types are marked as open squares, whereas those classified as AGN are marked as open circles. It seems clear that the red-fast objects are $\mathrm{SNe}$, whereas the blue-slow objects are AGN.

Of the five objects with spectra classified as AGN, one is noisy and has only a provisional classification; the other four show broad emission lines consistent with redshifts of $\mathrm{z}=0.43,0.89,1.00$, and 1.68 . These are all clearly larger than the plausible redshifts of the pre-existing SDSS galaxies. It seems clear then that actually we are seeing an event in a distant background AGN behind a foreground galaxy. As we discuss below, background AGN will occur behind foreground AGN reasonably often, but optical variability of this scale, 1-3 magnitudes, is essentially never seen (e.g. Schmidt et al. 2010, 2012).

\section{BRIGHT TRANSIENTS AS MICROLENSED AGN}

Our suggested explanation is that these slow-blue events are background AGN amplified by stellar micro-lensing in the foreground galaxy. Previous claims of quasar microlensing have involved ongoing low-level differential variability ( $\sim 0.3 \mathrm{mag})$ of multiple components of quasars that are also macro-lensed by the galaxy potential (Irwin et al. 1989; Eigenbrod et al. 2008; Morgan et al. 2010; Jimenez-Vicente et al. 2012). In our case, the

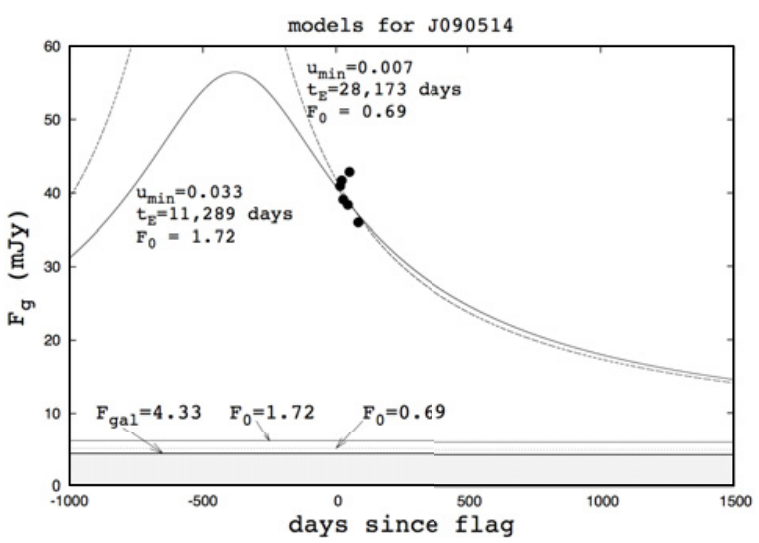

Figure 3. Light curve for J090514 fitted with simple microlensing models. The grey box indicates the flux level of the foreground SDSS galaxy. The solid and dotted lines just above this indicate two different possible levels for the background AGN before amplification. The model light curves shown correspond to these different base levels.

macro-lensing will be small (maybe 20\%) and we are talking about rare high amplitude events, dominated by a single star. Such a micro-lensing event has been previously proposed as one possible explanation for a flare in $\mathrm{a} z=2$ quasar seen behind M31 (Meusinger et al. 2010).

Fig. 3 shows simple single star microlensing models fitted to a typical object. The parameters are $F_{0}$, the flux level before amplification, $t_{E}$, the time to cross the Einstein radius $\theta_{E}$, and $u_{\min }=\theta_{\min } / \theta_{E}$, the impact parameter in terms of $\theta_{E}$. To a reasonable approximation for a high amplification event, the amplification $A=F_{\max } / F_{0}=\sim$ $1 / u_{\min }$ and the observed two-folding timescale is $t_{1 / 2} \sim$ $2 u_{\min } t_{E}$. The limited data we have so far gives a reasonable determination of $t_{1 / 2}$, but we do not know the value of $F_{0}$. This means that various different combinations of $F_{0}$ and $u_{\min }$ are possible, as indicated in Fig. 3. However, the probability of an event goes as $u_{\min }^{2}$. Larger $u_{\min }$ events are more likely, but an event with too large a value of $\mathrm{u}_{\min }$ implies a larger value of $F_{0}$, which would mean that the background AGN would already have been known. We can take as a minimalist model the one where the background AGN before amplification is 1 magnitude fainter than the foreground galaxy. In the example shown, this suggests an event with $u_{\min }=0.033$ and so $A=30$, and with a Einstein crossing timescale of 31 years, and thus an observed twofolding timescale of 2.0 years. Overall across our sample, it seems we are typically looking at events with $u_{\min } \sim$ $0.01-0.1$ i.e. with amplification factors $A=10-100$. Before amplification therefore, the background AGN are not quasar-like, but Seyfert like, with $L_{o p t} \sim 10^{43} \mathrm{erg} \mathrm{s}^{-1}$.

\section{IS THE MICROLENSING HYPOTHESIS REASONABLE?}

Extreme microlensing events must be rare. Does this hypothesis make sense? We are seeing AGN at $\sim 1$, foreground galaxies at $z \sim 0.2-0.3$, amplification $A \sim$ 30 , Einstein timescales of $10-100$ years, and around $10^{2}$ objects in "outburst" over the sky at any one time. How does this compare to what one might expect? 


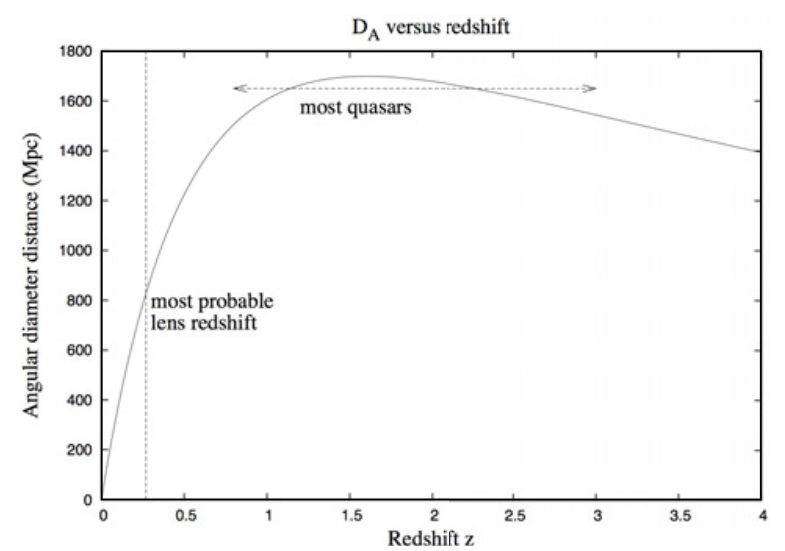

Figure 4. Behaviour of angular diameter distance versus redshift for a standard flat $\Lambda C D M$ model.

For a standard $\Lambda C D M$ cosmology, angular diameter distance comes to a broad flattish maximum at $z \sim 0.5-3$, fairly much the same redshift range containing most quasars - in other words from the point of view of lensing geometry, to a first approximation nearly all quasars are at roughly the same distance. (See Fig. 4). However, more distant AGN are fainter. As a result, we would expect most observed microlensed quasars to be at $z \sim 1$. The maximum lensing area per $d z$ shell is at $z \sim 0.25$. For the purposes of estimation, we can then take a typical event as having $z_{s}=1, z_{l}=0.25$ and $z_{l s}=0.6$.

The lens mass could of course have a wide range of possibilities. Small stars are more common, but the lensing area goes as $m^{2}$. Weighting the stellar mass function by $m^{2}$, the median lens mass is in fact close to $1 M_{\odot}$. Using this as a typical value, along with the typical redshift values above, we then get a characteristic size for the Einstein radius

$$
\theta_{E}=2.91 \mu \text { as }\left(M / M_{\odot}\right)^{1 / 2} \text {. }
$$

At the lens plane, this corresponds to a physical size of 2326AU. The relative motion of source and lens will be dominated by the relative motions of the two host galaxies. Taking $v=300 \mathrm{~km} \mathrm{~s}^{-1}$ as a reasonable value, the Einstein crossing time is then $t_{E}=$ 36.8 years $\left(M / M_{\odot}\right)^{1 / 2}(v / 300)^{-1}$. The observed timescale depends on the amplification, i.e. the impact parameter:

$$
t_{1 / 2}=893 \text { days }\left(M / M_{\odot}\right)^{1 / 2}(v / 300)^{-1}\left(u_{\min } / 0.033\right) .
$$

So the predicted timescale is in good agreement with what we see. What about the rate of events? If we consider a sightline through a galaxy like the Milky Way at $z_{l}=0.25$, then if we take the lens size to be $\theta_{E}$ for solar mass stars we get a covering factor of $f \sim 0.1$. This large value is why for known lensed quasars we do indeed see ongoing low-level flickering. However, for lens size given by $u_{\min }=0.033$ the covering factor is much smaller, $f \sim 10^{-4}$. As stars drift across the line of sight, how often do they cross the line of sight of the background AGN? The repeat time must be roughly

$$
t_{r p t} \sim 6000 \text { years }\left(M / M_{\odot}\right)^{1 / 2}(v / 300)^{-1}\left(u_{\min } / 0.033\right)^{-1} .
$$

However, not all foreground galaxies have a background AGN. For Seyfert-like luminosities, $L>10^{43} \mathrm{erg} \mathrm{s}^{-1}$, deep
Table 1. Outline of expected resolution effects.

\begin{tabular}{ccc}
\hline & $\begin{array}{c}\text { A=10 } \\
\text { lens 291nas }\end{array}$ & $\begin{array}{c}\text { A=100 } \\
\text { lens 29nas }\end{array}$ \\
\hline disc 12nas & $\begin{array}{c}\text { point sources } \\
\text { decline grey }\end{array}$ & $\begin{array}{c}\text { significant resolution effects } \\
\text { decline reddens }\end{array}$ \\
BLR 1200nas & $\begin{array}{c}\text { amplified less } \\
\text { weak lines }\end{array}$ & $\begin{array}{c}\text { amplified much less } \\
\text { featureless at peak }\end{array}$ \\
\hline
\end{tabular}

X-ray surveys find an AGN roughly once every square arcminute (e.g. Xue et al. 2011). So very roughly speaking, about $0.03 \%$ of galaxies have a suitable background AGN and about $0.05 \%$ of these are in outburst at any one time. The total number of galaxies around the sky at $\mathrm{g}=22$ is $\sim 10^{8}$. So in very rough terms, the number of microlensed flares active around the sky at any one time must be around 100 - very close to what we have found.

\section{FUTURE PROSPECTS}

To confirm the micro-lensing hypothesis, we need to continue and improve our light curves, and obtain more spectroscopic data. Following the fading transients over several years will be crucial to establish the preamplification flux, and so remove the degeneracy in light curve fitting, and find the distribution of amplification factors. We also need to model the light curves using galaxy simulations - in the analysis presented for this conference paper we have used a simple single-star model, whereas even at small impact parameter the effect of many overlapping stars will be significant (see Schmidt and Wambsganss 2010).

The microlensing phenomenon is more than a curiosity. It could provide a powerful technique for decoding the structure of accretion discs and the Broad Line Region (BLR). This is because we expect there will be significant resolution effects. For Seyfert-like objects, the AGN black hole has perhaps $M_{H} \sim 10^{8} M_{\odot}$. The accretion disc might be expected to to have a size $\sim 10 R_{S}$, but with size varying with wavelength. The Broad Line Region (BLR) will have a size of $\sim 1000 R_{S}$. At $z=1$ we then have $\theta_{\text {disc }}=12 R_{10} M_{8}$ nas whereas the lens size is $\theta_{\text {lens }}=291\left(u_{\text {min }} / 0.1\right)$ nas.

The nature of the resolution effects we should seen will then depend on the amplification factor of the event, as indicated in Table 1. For low amplification events, the continuum light curve should be grey, but we should see that BLR lines have on average lower equivalent widths than usual. For high amplification events, the continuum colours should change during the event, and the continuum will be amplified much more than the BLR, so that near peak we may see an apparently featureless continuum, with broad lines appearing during the decline.

Related resolution effects have already been claimed for multiple macro-lensed quasars, but of a more modest size, and of a statistical rather than systematic nature. In the optical, such studies have suggested that the observed size of quasars is a factor $\sim 5$ larger than expected from accretion disc theory (Morgan et al. 2010; Mosquera 
et al. 2011), while the X-ray source is smaller than the optical source (Dai et al. 2010), and the BLR has, as expected, smaller variations (Lewis et al. 1998; Sluse et al. 2011). However, monitoring systematic trends in individual objects, and looking for the expected differences with amplification factor, will be much cleaner and more powerful.

Finally, we may ask ourselves, if by looking for TDEs we have found microlensing effects, how do we distinguish the true TDEs? Amongst the events we have seen, they could perhaps be the ultra-blue objects, or the blue-butfast objects. However, those could also be the highestamplification lensing events - they will be the "peakiest", and the blue light from the AGN may well be more strongly amplified than the red light. The key to separating TDEs from microlenisng effects will be getting a clear redshift for both the transient event and the host galaxy; for a TDE these should be the same.

This work builds on the hard work of the PanSTARRs project team, especially the pipeline team, and the PS1 Science Consortium formed to exploit PanSTARRS-1. In particular, we rely on the Belfast system for filtering, classifying and organising the transient stream.

\section{References}

[1] Dai, X, et al, ApJ 709, (2010) 278-285

[2] Eigenbrod, A, et al, A\&A 490, (2008), 933-943

[3] Evans, CR \& Kochanek, CS, ApJ 346, (1989), L13L16

[4] Gezari, S, et al Nature 485, (2012), 217-220

[5] Irwin, M, et al ApJ 98, (1989), 1989-1994

[6] Jimenez-Vicente, J, et al arXiv 1201, (2012), 1201.3187

[7] Kaiser, N, et al, SPIE 7733, (2010), 77330E-77330E14

[8] Lewis, GF, et al, MNRAS 195, (1998), 573-586

[9] Lodato, G, et al, MNRAS 392, (2009), 332-340

[10] Meusinger, H, et al A\&A 512, (2010), A1

[11] Morgan, C, et al, ApJ 712, (2010), 1129-1136

[12] Mosquera, A, et al, ApJ 728, (2011), 145

[13] Richards, G, et al, AJ 121, (2001), 2308-2330

[14] Schmidt, R, and Wambsganss, J, Gen.Rel. and Gravitation 42, (2010), 2127-2150

[15] Schmidt, K, et al, ApJ 714, (2010), 1194-1208

[16] Schmidt, K, et al, ApJ 744, (2012), 147

[17] Sluse, D, et al, A\&A 528, (2011), A100

[18] Xue, Y, et al, ApJSupp 195, (2011), 10 